\title{
With thanks to our 2018 peer reviewers
}

We are grateful to the following people for their significant contribution to Health Promotion and Chronic Disease Prevention in Canada as peer reviewers in 2018. Their expertise ensures the quality of our journal and promotes the sharing of new knowledge among peers in Canada and internationally.

\begin{tabular}{|c|c|c|}
\hline Ashley Aimone & Tara Gomes & Catherine Pelletier \\
\hline Philippe Autier & Andrea Gruneir & Paul Peters \\
\hline Juanita Bacsu & Heather Hannah & Sarah A. Richmond \\
\hline Farin Bakhtiari & Lisa D. Hawke & Nancy A. Ross \\
\hline Maulik Baxi & Katelyn Holliday & Tayyab Shah \\
\hline Patrick Berrigan & Birgit Isernhagen & Michèle Shemilt \\
\hline Jennifer Bethell & Shanthi Johnson & Kelley J. Sittner \\
\hline Monique Bordeleau & Tarun Katapally & Amanda K. Slaunwhite \\
\hline Songul Bozat-Emre & Scott Kehler & Richard S. Stanwick \\
\hline Ruben Brondeel & Soyeon Kim & Mark C.J. Stoddart \\
\hline Lauren Burt & Melissa Kimber & Carol Strike \\
\hline Yue Chen & Rachel Laxer & Mariano Suppa \\
\hline Phil Chilibeck & Eun-Young Lee & Samuel Tomczyk \\
\hline Susan Clayton & Shelly-Anne Li & Linda Varangu \\
\hline Erica Di Ruggiero & Alyson Mahar & Paul Villeneuve \\
\hline Carolyn Dohoo & John McLaughlin & Daniel Warshafsky \\
\hline Frank J. Elgar & Jacqueline Middleton & Rania A. Wasfi \\
\hline Tara Elton-Marshall & Elaine Moody & Colleen Whyte \\
\hline Philippe Finès & Patrick Morency & Michel D. Wissing \\
\hline Jean-Michel Galarneau & Howard Morrison & Niko Yiannakoulias \\
\hline Fidji Gendron & Karen A. Patte & Jennifer Zwicker \\
\hline
\end{tabular}

